\title{
Comparison of Magnetic Domain Wall Images using Lorentz Microscopy and Magnetic Force Microscopy
}

\author{
S. Hua and M. De Graef \\ Department of Materials Science and Engineering, Carnegie Mellon Univ., Pittsburgh PA 15213
}

Magnetic domain walls play a central role in the energetics of magnetic materials, and a direct experimental measurement method of their width would be an extremely valuable tool [1]. Theoretical studies of domain wall profiles in ferromagnetic thin films have been carried out in terms of a balance between the quantum mechanical exchange stiffness and any anisotropy present [2]. Several wall width measurement procedures have been proposed using either Fresnel mode Lorentz TEM or differential phase contrast electron microscopy [3-5]. However, there is still a discrepancy between experimental and theoretical domain wall widths (DWWs) due to different limitations. Recently, the Transport-ofIntensity Equation approach [6], based on Fresnel imaging, has provided a new method to map the inplane magnetic induction, from which an estimate of the domain wall width can be extracted [7].

All Fresnel image acquisition of DWWs was performed on a 200kV FEI Tecnai F20 TEM in Lorentz mode. To carry out the phase reconstruction, a set of in-focus, under-focus and over-focus Fresnel images with equidistant defocus values should be obtained. Then, the phase shift gradient can be calculated by the TIE formalism from an aligned image set. The slope of the phase shift is constant within a uniformly magnetized domain, whereas it changes continuously across the domain wall. Thus, the range of this change can be used to define the DDW. Fig. 1 shows preliminary wall width measurements for two kinds of magnetic thin films: $\mathrm{Ni}_{50} \mathrm{Mn}_{27} \mathrm{Ga}_{23}$ and $\mathrm{Fe}_{70} \mathrm{Ni}_{30}$; the color map on the left shows the magnetic domain configuration; the phase shift profiles across the indicated domain walls are shown on the right. The average domain wall width measurement results are $17.5 \mathrm{~nm}$ for $\mathrm{Ni}_{50} \mathrm{Mn}_{27} \mathrm{Ga}_{23}$ and $26.5 \mathrm{~nm}$ for $\mathrm{Fe}_{70} \mathrm{Ni}_{30}$. In general, this measurement approach shows good agreement with theoretical wall width calculations and is easier to carry out than the traditional technique which involves extrapolation of the width of the dark domain wall Fresnel image to zero defocus.

Because the effect of applied stress on magnetic domain configurations can be significant, it is of interest to determine how the magnetic phase shift changes with applied stress. The magnetization state of a pair of $180^{\circ}$ domain walls was determined using an energy minimization which includes magnetoelastic anisotropy. Fig. 2 represents the Laplacian of the phase shift across a domain wall of a Fe thin film with zero, compressive and tensile stress applied; the DWW change as a function of applied stress is clearly. However, it is difficult to apply a stress to a TEM thin foil. Magnetic force microscopy (MFM) has been widely exploited to study surface magnetic-stray-fields. Some types of MFM provide an open sample stage suitable for in-situ observations. This contribution will report on a direct comparison of DWWs as measured by LTEM and MFM, including the simulation of the MFM images.

\section{References}

[1] De Graef, M., Y. Zhu, Magnetic imaging and its applications to materials, in Experimental Methods in the Physical Sciences, 2001, Academic Press.

[2] Hubert, A., R. Schäfer, Magnetic domains: the analysis of magnetic microstructures, 1998, Springer.

[3] Suzuki, T., C.H. Wilts, C.E. Patton, 1983. J. Appl. Phys. 39(1983).

[4] J. Marti, W.F. Lewis, 1977. J. Appl. Phys. 48, 3167 (1977). 
[5] G. R. Morrison, H. Gong, J. N. Chapman, and V. Hrnciar. J. Appl. Phys. 48, 3167 (1977).

[6] M. Beleggia, M.A. Schofield, V.V. Volkov, Y. Zhu. Ultramicroscopy. 102. 37-39(2004).

[7] De Graef, M., Introduction to conventional transmission electron microscopy, 2003, Cambridge.

[8] The authors acknowledge funding from the National Science Foundation, DMR-10005330.
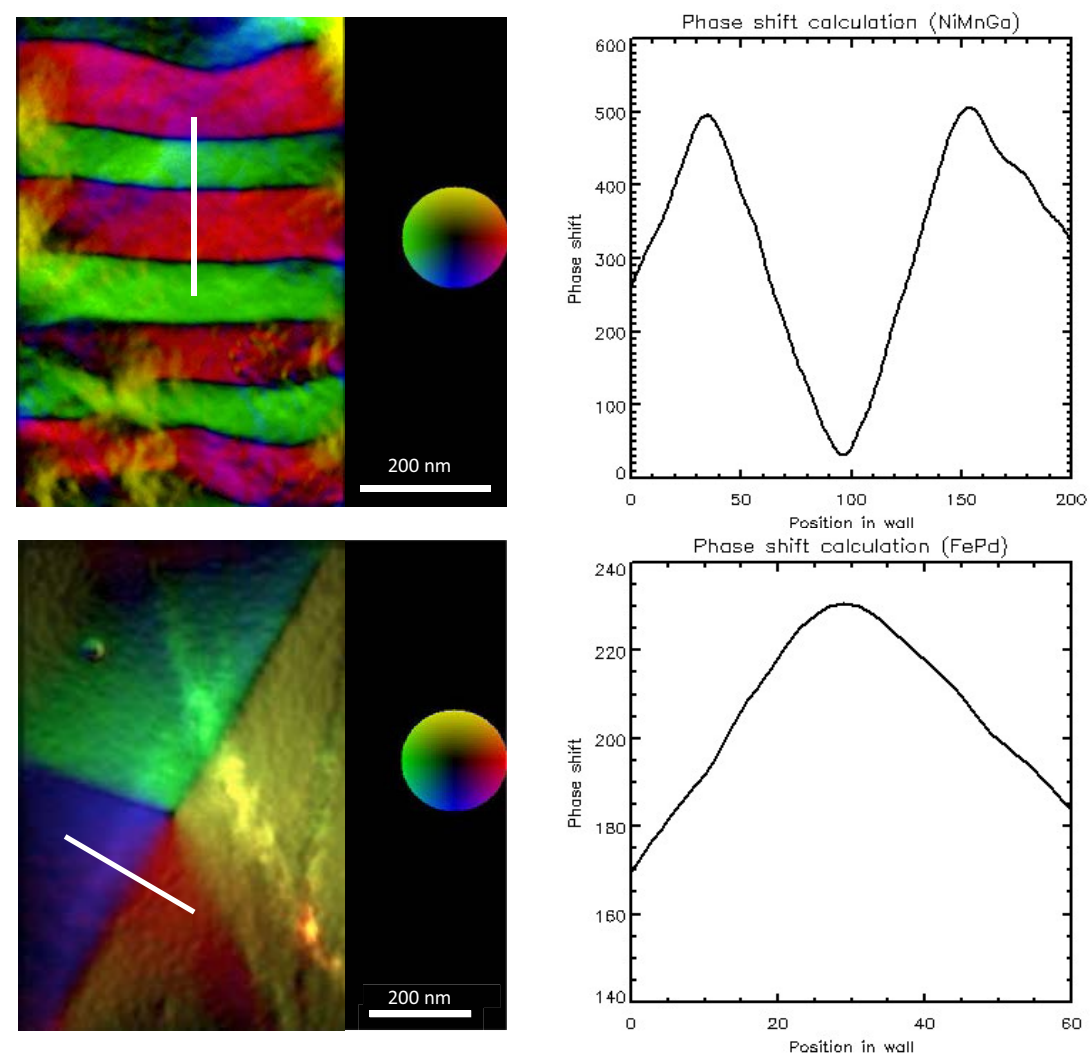

Figure 1: Magnetic contrast color map of several parallel 180 。 domain walls in a $\mathrm{Ni}_{50} \mathrm{Mn}_{27} \mathrm{Ga}_{23}$ (top row) and $\mathrm{Fe}_{70} \mathrm{Ni}_{30}$ (bottom row) (left); phase shift (non-calibrated) profile across three $180 \circ$ domain walls extracted from phase reconstructions.
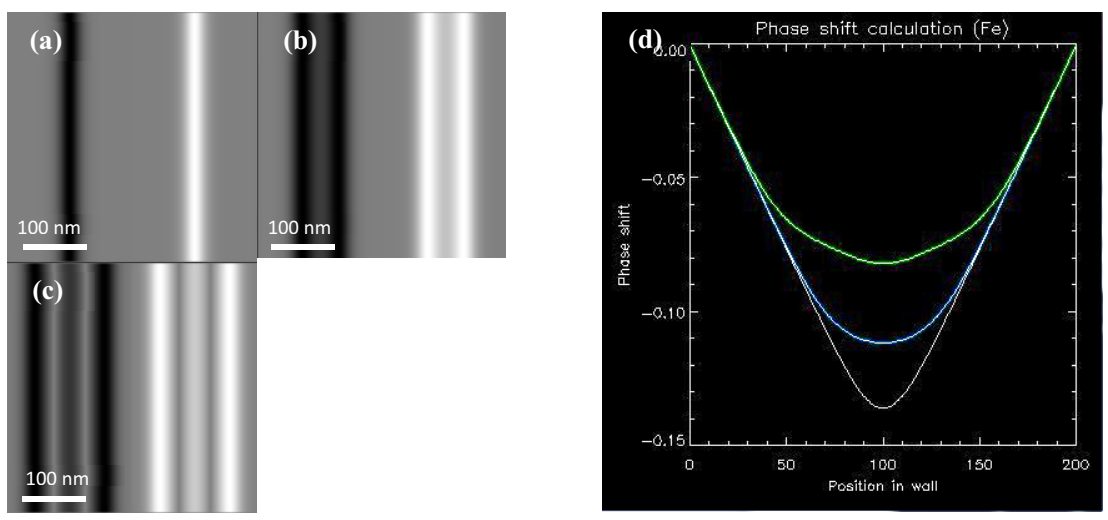

Figure 2: Laplacian of the phase shift map of two 180॰ domain wall for cubic crystal pure iron (a) (c), under zero stress (a), tensile (b) and compressive (c) stress; Plot of phase shift profile in the direction of wall normal (d), green - tensile stress, blue - compressive stress, white - without stress. 